\title{
On a generalization of Mycielski's and Znám's conjectures about coset decomposition of Abelian groups
}

by

\author{
Ivan Korec (Bratislava)
}

Abstract. An exact lower bound for the cardinality of a partition of a group into cosets by its invariant subgroups is given. This lower bound is a function of the index of the intersection of all the subgroups, cosets by which occur in-the partition. For Abelian groups the present theorem gives the bound conjectured by Mycielski and Sierpiński, and for the infinite cyclic group we obtain the bound conjectured by Znám.

In this paper groups will be considered as multiplicative groups and their neutral element will be denoted by $e$. The order of a finite group $G$ will be denoted by $|G|$. If $H$ is a subgroup of $G$ and $a \epsilon G$, then $[G: H]$, $a H$ will denote the index of $H$ in $G$ and the set $\{a h \mid h \in H\}$, respectively. If $H$ is an invariant subgroup of $G$, then $G / H$ will denote the factor group of $G$ by $H$. In the notation of the form $\left\{x_{1}, \ldots, x_{n}\right\}$ we always suppose $x_{1}, \ldots, x_{n}$ to be pairwise different.

J. Mycielski and W. Sierpiński [2] made the following conjecture: Let

$$
\left\{a_{1} G_{1}, a_{2} G_{2}, \ldots, a_{k} G_{k}\right\}
$$

be a coset decomposition of an Abelian groap $G$ (i.e., the elements of (1) are pairwise disjoint cosets of $G$ and its set-theoretical union is $G$ ) and let $n=\left[G: G_{1}\right]$ be finite. If

$$
n=\prod_{i=1}^{r} p_{i}^{a_{i}}
$$

is the standard form of $n$, then

$$
k \geqslant 1+\sum_{i=1} \alpha_{i}\left(p_{i}-1\right) .
$$

S. Znam made a similar conjecture in which $G$ is the additive group of integers and the condition $n=\left[G: G_{1}\right]$ is replaced by $n=\left[G: \bigcap_{i=1}^{k} G_{i}\right]$, but the proof was not published. 
Theorem 1 of the present paper is a generalization of both conjectures above.

For every (non-zero) natural $n$ with the standard form (2) put

$$
\mathscr{F}(n)=\sum_{i=1}^{r} \alpha_{i}\left(p_{i}-1\right)
$$

LEMMA 1. For arbitrary non-zero naturals $m, n$

$$
\mathcal{F}(m n)=\mathscr{F}(m)+\mathscr{F}(n),
$$$$
F(n)<n \text {. }
$$

Proof. (5) is obviously true. If $n$ is a prime or $n=1$, then $F(n)$ $=n-1<n$. Let $(6)$ hold for all $n$ which are products of at most $k$ primes, and let $m$ be a product of $k+1$ primes. Suppose $m=p n$ where $p$ is a prime. Then

$\mathscr{F}(m)=\mathscr{F}(p)+\mathscr{F}(n) \leqslant(p-1)+(n-1) \leqslant(p-1) n+(n-1)<m, \quad$ q.e.d.

Definition. Let $G$ be a (not necessary Abelian) group. (1) will be called an invariant coset decomposition (IOD) of the group $G$ if all the groups $G_{1}, G_{2}, \ldots, G_{k}$ are invariant subgroups of $G$ and if every element of $G$ belongs to exactly one element of (1).

The main result of this paper is:

THEOREM 1. Let $G$ be a group and (1) its IOD. Then

$$
k \geqslant 1+\mathscr{F}\left(\left[G: \bigcap_{i=1}^{k} G_{i}\right]\right)
$$

Remark. In the following we show the finiteness of $\left[G: \bigcap_{i=1}^{k} G_{i}\right]$. Theorem 1 is obviously a generalization of Znám's conjecture. It is also a generalization of Mycielski's conjecture because every coset decomposition of an Abelian group $G$ is its ICD, and $F\left(\left[G: \bigcap_{i=1}^{k} G_{i}\right]\right) \geqslant F\left(\left[G: G_{1}\right]\right)$.

Limma 2. Let $G$ be a group and $a_{1} G_{1}, a_{2} G_{2}$ its cosets. Then either $a_{1} G_{1} \cap$ $\cap a_{2} G_{2}=\varnothing$ or $a_{1} G_{1} \cap a_{2} G_{2}$ is a coset by $G_{1} \cap G_{2}$.

Proof. Suppose $a_{1} G_{1} \cap a_{2} G_{2} \neq \varnothing$ and $b \in a_{1} G_{1} \cap a_{2} G_{2}$. Then $a_{1} G_{1}$ $=b G_{1}, a_{2} G_{2}=b G_{2}$ and hence $a_{1} G_{1} \cap a_{2} G_{2}=b\left(G_{1} \cap G_{2}\right)$, q.e.d.

LEMMA 3. If $G$ is a group, $x \in G$ and (1) is an IOD of $G$, then

$$
\left\{\left(x a_{1}\right) G_{1},\left(x a_{2}\right) G_{2}, \ldots,\left(x a_{k}\right) G_{k}\right\}
$$

is also an ICD of the group $G$.

The proof is obvious.

LEMMA 4. Let (1) be an IOD of a group G. Let $F$ be an invariant subgroup of $G$ contained in $\bigcap_{i=1}^{k} G_{i}$. Write $\bar{G}=G / F, \bar{G}_{i}=G_{i} / F$ and $\bar{a}_{i}=a_{i} F$ for every $i=1,2, \ldots, k$. Then

is an IOD of the group $\bar{G}$ and $\left[\bar{G}: \bigcap_{i=1}^{k} \bar{G}_{i}\right]=\left[G: \bigcap_{i=1}^{k} G_{i}\right]$.

Proof. By Theorem 2.3.2 and Theorem 2.3.4 of [1] $\bar{G}_{1}, \bar{G}_{2}, \ldots, \bar{G}_{k}$ are invariant subgroups of $\bar{G}$. Denote by $\varphi$ the canonical homomorphism from $G$ onto $\bar{G}$. Since, for every $i=1,2, \ldots, k, \varphi^{-1}\left(\bar{a}_{i} \bar{G}_{i}\right)=a_{i} G_{i}$ and $(1)$ is from $G$ onto $G$. Since, for every $i=1,2, \ldots, k, \varphi^{-1}\left(\bar{a}_{i} G_{i}\right)=a_{i} G_{i}$ partition of $G,(9)$ is a partition of $\bar{G}$. Since $\varphi^{-1}\left(\bigcap_{i=1}^{k} \bar{G}_{i}\right)=\bigcap_{i=1}^{k} G_{i}$, we have $\left[\bar{G}: \bigcap_{i=1}^{k} G_{i}\right]=\left[G: \bigcap_{i=1}^{k} G_{i}\right]$.

LEMima 5. Let $G$ be a group and (1) its ICD. Then $\left[G: \bigcap_{i=1}^{k} G_{i}\right]<\infty$ and $\left[G: G_{i}\right]<\infty$ for every $i=1,2, \ldots, k$.

Proof. Since every coset by $\bigcap_{i=1}^{k} G_{i}$ is an intersection of some cosets by $G_{i}$, we have $\left[G: \bigcap_{i=1}^{k} G_{i}\right] \leqslant \prod_{i=1}^{k}\left[G: G_{i}\right]$. Hence it is sufficient to prove $\left[G: G_{i}\right]<\infty$ for $i=1_{i=1}^{1}, 2, \ldots, k_{i=1}$. Let $k$ be the smallest natural number for which there exists a group $G$ and an IOD (1) of $G$ such that [G: $\left.G_{r}\right]$ is infinite for some $r=1,2, \ldots, k$.

Suppose at first that $\left[G_{i}: G_{i} \cap G_{j}\right]$ is infinite for some $i, j=1,2, \ldots, k$. By Lemma 3 without loss of generality $a_{j}=e$ can be assumed. Then the non-empty elements of the sequence

$$
G_{i} \cap a_{1} G_{1}, G_{i} \cap a_{2} G_{2}, \ldots, G_{i} \cap a_{k} G_{k}
$$

form an IOD of $G_{i}$ with less than $k$ elements (because $G_{i} \cap a_{i} G_{i}=\varnothing$ ) and with at least one infinite index $\left[G_{i}: G_{i} \cap G_{j}\right]$. This contradiction implies that all the indices $\left[G_{i}: G_{i} \cap G_{j}\right]$ are finite.

$$
\begin{array}{r}
\text { Denote } \bigcap_{i=1}^{k} G_{i} \text { by } F . \text { For every } s=1,2, \ldots, k \text { we have } \\
{\left[G_{s}: F\right]=\left[G_{s}: \bigcap_{i=1}^{k} G_{s} \cap G_{i}\right] \leqslant \prod_{i=1}^{k}\left[G_{s}:\left(G_{s} \cap G_{i}\right)\right]}
\end{array}
$$

and hence all the indices $\left[G_{s}: F\right]$ are finite. Every coset $a_{i} G_{i}$ consists of $\left[G_{i}: F\right]$ cosets by $F$ and hence the group $G$ consists of $[G: F]=\sum_{i=1}^{k}\left[G_{i}: F\right]$ cosets by $F$. However, $\left[G: G_{i}\right] \leqslant[G: F]$ for every $i=1,2, \ldots, k$, and hence all the indices $\left[G: G_{i}\right]$ are finite, which is a contradiction.

Remark. Lemma 5 is a corollary of Theorem 1 of [4]. That paper has not been published yet. 
LEMMA 6. Let $G$ be a group, let (1) be IOD of $G$ and let $H$ be a (proper) maximal invariant subgroup of $G$. Then

a) every element of (1) either is contained in some member of $G / H$ or has a non-empty intersection with every element of $G / H$.

b) if an element of (1) is contained in some element of $G / H$, then every element of $G / H$ contains a number of (1).

Proof. a) It is sufficient to consider $a_{1} G_{1}$, and without loss of generalitry $a_{1}=e$ can be supposed. Consider the set-theoretical union of all members of $G / H$ which have a non-empty intersection with $G_{1}$, and denote it by $F$. Obviously $F=G_{1} H=\left\{g h \mid g \in G_{1}, h \in H\right\}$. By [1], Theorem 2.3.3, $F$ is an invariant subgroup of $G$. Since $H \subseteq F \subseteq G$, we have either $F=H$ or $F=G$. If $F=H$, i.e. $G_{1} H=H$, then $G_{1} \bar{C} H$. Let $G_{1} H=G$ and let $x$ be an arbitrary element of $G$. There exist $\bar{y} \epsilon G_{1}, z \in H$ such that $x=y z$. Hence $G_{1} \cap x H=G_{1} \cap y z H=y G_{1} \cap y H=y\left(G_{1} \cap H\right) \neq \varnothing$, q.e.d.

b) Let an element of (1) be contained in some element of $G / H$. Denote $\bigcap_{i=1}^{k} G_{i}$ by $F$. Obviously $F \subseteq H$ holds. By Lemma $5,[G: F]$ is finite. Suppose that $\left\{a_{1} G_{1}, a_{2} G_{2}, \ldots, a_{r} G_{r}\right\}$ is the set of all elements of (1) which have a non-empty intersection with every member of $G / H$. Every intersection $a_{i} G_{i} \cap x H(i=1,2, \ldots, r, x \in G)$ consists of $\left[H \cap G_{i}: F\right]$ elements of $G / F$. Hence $\left(\bigcup_{i=1}^{r} a_{i} G_{i}\right) \cap x H$ consists of $\sum_{i=1}^{r}\left[H \cap G_{i}: F\right]$ elements of $G / H$, and then $x H-\left(\bigcup_{i=1}^{r} a_{i} G_{i}\right)$ consists of $\left[H: F^{\prime}\right]-\sum_{i=1}^{r}\left[H \cap G_{i}: F^{\prime}\right]$ elements of $G / F$. This number does not depend on $x$. Since it is non-zero for some $x \in G$, it is non-zero for every $x \in G$, i.e. every set $x H-\left(\bigcup_{i=1}^{r} a_{i} G_{i}\right)$ contains at least one coset by $F$ (as a subset). This coset must be contained in some $a_{j} G_{j}, j>r$. By a), $a_{j} G_{j}$ is a subset of $x H$, q.e.d.

Proof of Theorem 1. In the sequel, a "group" means an invariant subgroup of $G$ and a "coset" means a coset of $G$ by an invariant subgroup of $G$. By Lemma 4 and Lemma 5 it is sufficient to consider the case $|G|<\infty$ and $\left|\bigcap_{i=1}^{k} G_{i}\right|=1$. Now we can prove Theorem 1 by induction with respect to $|G|^{i=1}$.

If $|G|=1$ then $k=1, \bigcap_{i=1}^{k} G_{i}=G$, and (7) obviously holds.

Let $|G|>1$. Denote by $\mathfrak{M}$ the set of all such non-empty subsets $M$ of $\{1,2, \ldots, k\}$ that there exists a partition

$$
\left\{X_{1}, X_{2}, \ldots, X_{m}\right\}
$$

of the group $G$ so that every element of (1) is contained in a member of (11), every coset by $\bigcap G_{i}$ is also contained in a member of (11) and

$$
i \in M
$$

$$
m \geqslant 1+\mathcal{F}\left(\left[G: \bigcap_{i \in M} G_{1}\right]\right) .
$$

Suppose now $\{1,2, \ldots, k\} \in \mathfrak{M}$. Then there exists such a partition (11) of $G$ that $m \geqslant 1+\mathscr{F}\left(\left[G: \bigcap_{i=1}^{k} G_{i}\right]\right)$. However, $k \geqslant m$ and hence (7) holds.

Therefore to finish the proof of Theorem 1 it suffices to show that $\{1,2, \ldots, k\} \in \mathfrak{M}$.

Proposition A. The set $\mathfrak{M}$ is non-empty.

Without loss of generality $a_{1}=e$ can be put. Since $G_{1}$ is a proper subgroup of $G$, there exists a maximal proper subgroup $H$ of $G$ containing $G_{1}$. The non-empty members of the sequence

$$
H \cap a_{1} G_{1}, H \cap a_{2} G_{2}, \ldots, H \cap a_{k} G_{k}
$$

form an ICD of the group $H$ because $H \cap G_{1}, H \cap G_{2}, \ldots, H \cap G_{k}$ are invariant subgroups of $H$. We may suppose that

$$
H \frown a_{1} G_{1}, H \frown a_{2} G_{2}, \ldots, H \frown a_{s} G_{s}
$$

are non-empty and that the other members of (13) are empty. We have $|H|<|G|$ and hence, by the induction hypothesis,

$$
s \geqslant 1+\mathcal{F}\left(\left[H: \bigcap_{i=1}^{s}\left(H \cap G_{i}\right)\right]\right)=1+\mathcal{F}\left(\left[H: \bigcap_{i=1}^{s} G_{i}\right]\right) .
$$

Let $[G: H]=h$ and $G / H=\left\{H^{1}, H^{2}, \ldots, H^{h}\right\}, H^{1}=H$. Consider the set

$$
\left\{a_{1} G_{1}, a_{2} G_{2}, \ldots, a_{s} G_{s}, H^{2}-\bigcup_{i=1}^{s} a_{i} G_{i}, \ldots, H^{h}-\bigcup_{i=1}^{s} a_{i} G_{i}\right\} .
$$

The elements of (16) are obviously pairwise disjoint and their union is the set $G$. Clearly $a_{1} G_{1} \subseteq H$ and hence by Lemma 6 every coset $H^{j}, j=2, \ldots, h$, also contains an element of (1). Therefore the sets $H^{j}-\bigcup_{i=1}^{s} a_{i} G_{i}, j=2, \ldots, k$, are non-empty, and hence (16) is a partition of the group G. By Lemma 6 every member of (1) either has a non-empty intersection with every element of $G / H$ or is a subset of some $H^{j}$, $j=1,2, \ldots, h$. Hence every element of (1) is contained in an element of (16). It is easy to see that every coset by $\bigcap_{i \in M} G_{i}$ is also contained in some element of (16). To prove $\{1,2, \ldots, s\} \in \mathfrak{M} \mathfrak{i \in M}$ it remains to verify that $s+h-1 \geqslant 1+\mathfrak{F}\left(\left[G: \bigcap_{i=1}^{s} G_{i}\right]\right)$. 
By Lemma 1 we have

$$
\begin{aligned}
s+h-1 & \left.\geqslant 1+\mathscr{F}\left(\left[H: \bigcap_{i=1}^{s} G_{i}\right]\right)+[G: H]\right)-1 \geqslant 1+\mathscr{F}\left(\left[H: \bigcap_{i=1}^{s} G_{i}\right]\right)+\widetilde{F}^{\tau}([G: H]) \\
& =1+\mathscr{F}\left([G: H]\left[H: \bigcap_{i=1}^{s} G_{i}\right]\right)=1+\mathscr{F}\left(\left[G: \bigcap_{i=1}^{s} G_{i}\right]\right), \quad \text { q.e.d. }
\end{aligned}
$$

Proposimion B. No proper subset of $\{1,2, \ldots, k\}$ is a maximat element of $\mathfrak{M}$.

Assume on the contrary that $M \neq\{1,2, \ldots, k\}$ is a maximal element of $\mathfrak{M}$. Without loss of generality $M=\{s, s+1, \ldots, l\}\}$ and $a_{1}=e$ can be assumed. Let (11) be a partition of $G$ such that every element of (1) is contained in some element of (11), every coset by $\cap G_{i}$ is contained in some element of (11) and let (12) hold. Put $F \stackrel{\bigcap_{i \in M}}{G_{i}} G_{i}$ Obviously $F \cap a_{i} G_{i}=\varnothing$ for $i \in M$ and hence the non-empty members of the sequence

$$
F \cap a_{1} G_{1}, F \cap a_{2} G_{2}, \ldots, F \cap a_{s-1} G_{s-1}
$$

form a partition of the gronp $F$. We may assume that

$$
F \cap a_{1} G_{1}, F \cap a_{2} G_{2}, \ldots, F \cap a_{r} G_{r}
$$

are non-empty and that the other members of (17) are empty. Clearly $|F|<|G|$ and hence by Lemma 2 and the inductive hypothesis

$$
r \geqslant 1+\mathscr{F}\left(\left[F: \bigcap_{i=1}^{r}\left(F \cap G_{1}\right)\right]\right) .
$$

The group $F$ is contained in some member of (11); suppose $F \subseteq X_{m}$. Then every coset $a_{i} G_{i}, i=1,2, \ldots, r$, is also contained in $X_{m}$ becanse it is a subset of some member of (11) and has a non-empty intersection. with $F$. Consider now the set

$$
\left\{a_{1} G_{1}, a_{2} G_{2}, \ldots, a_{r} G_{r}, X_{1}, X_{2}, \ldots, X_{m-1}, X_{m}-\bigcup_{i=1}^{r} a_{i} G_{i}\right\} .
$$

Let $N=M \cup\{1,2, \ldots, r\}$. Every coset by $\bigcap_{i \in N} G_{i}=\left(\bigcap_{i \in M} G_{i}\right) \cap F$ is contained in some element of $(20)$. Every element of $(1)$ is also contained in some element of (20). The number of non-empty elements of (20) is. at least $r+m-1$ and we have

$$
\begin{aligned}
r+m-1 & \left.\geqslant 1+\mathscr{F}\left(\left[F: \bigcap_{i=1}^{r}\left(F \cap G_{i}\right)\right]\right)+\mathscr{F}\left(\left[G: F^{\prime}\right]\right)\right]=1+F\left(\left[G: \bigcap_{i=1}^{r}\left(F \cap G_{i}\right)\right]\right) \\
& =1+\mathscr{F}\left(\left[G:\left(\bigcap_{i=1}^{r} G_{i} \cap \bigcap_{i \in M} G_{i}\right)\right]\right)=1+\mathscr{F}\left(\left[G: \bigcap_{i \in N} G_{i}\right]\right) .
\end{aligned}
$$

Hence $N \in \mathfrak{M}$, contrary to the maximality of $M$.
Propositions A and B imply $\{1,2, \ldots, k\} \in \mathfrak{M}$. Therefore (7) holds, q.e.d.

Actually we have proved more than Theorem 1. For an arbitrary group $G$ and its invariant subgroup $F^{\prime}$ such that $\left[G: F^{\prime}\right]<\infty$ define

$$
\Im(G, F)=\sum_{i=1}^{s}\left(\left[H_{i}: H_{i-1}\right]-1\right),
$$

where $H_{0}=F, H_{1}, \ldots, H_{8}=G$ is a maximal chain of subgroups of $G$ such that $H_{i}$ is invariant in $H_{i+1}$ for all $i=0,1, \ldots, s-1$. By [1], Theorem 8.4.4, $\mathcal{G}(G, F)$ does not depend on the choice of the chain $H_{0}, H_{1}, \ldots$ $\ldots, H_{\delta}$. The following theorem can be proved in the same way as Theorem 1:

THEOREM 2. Let $G$ be a group and (1) its IOD. Then

$$
k \geqslant 1+\mathcal{G}\left(G, \bigcap_{i=1}^{k} G_{i}\right) .
$$

If the group $G$ is Abelian, then the right sides of (7) and (22) are equal. However, there are (non-Abelian) groups and their IOD (1) for which the right side of (22) is greater than that of (7).

Remark. Theorem 1 does not hold if we replace the words "invariant coset decomposition" by the "left coset decomposition" or "right coset decomposition". We give some examples. Let $G=S_{3}$ be the symmetric group of degree $3, S_{2}$ one of its two-elements subgroups and $A_{1}, A_{2}, A_{3}$ (resp. $B_{1}, B_{2}, B_{3}$ ) right (resp. left) cosets of $S_{3}$ by $S_{2}$. If we consider the set $\left\{A_{1}, A_{2}, A_{3}\right\}$ (resp. $\left.\left\{B_{1}, B_{2}, B_{3}\right\}\right)$ as a left (resp. right) coset decomposition $\left\{a_{1} G_{1}, a_{2} G_{2}, a_{3} G_{3}\right\}$ (resp. $\left\{G_{1} a_{1}, G_{2} a_{2}, G_{3} a_{3}\right\}$ ) of the group $G$, then $G_{1} \cap G_{2} \cap G_{3}=\{e\}$. We have $k=3$ and $1+\mathscr{F}([G:\{e\}])$ $=1+\mathscr{F}(6)=4$. Hence $(7)$ does not hold.

We give one more example. If we consider

$$
\left\{A_{1} \times B_{1}, A_{2} \times B_{1}, A_{3} \times B_{1}, S_{3} \times B_{2}, S_{3} \times B_{3}\right\}
$$

as a left (resp. right) coset decomposition $\left\{a_{1} G_{1}, \ldots, a_{5} G_{5}\right\}$ (resp. $\left\{G_{1} a_{1}, \ldots\right.$ $\left.\left.\ldots, G_{5} a_{5}\right\}\right)$ of the group $G=S_{3} \times S_{3}$, then $G_{3} \cap G_{2} \cap \ldots \cap G_{5}=S_{2} \times\{e\}$ (resp. $\{e\} \times S_{2}$ ). In both cases $t=5$ and the right side of $(7)$ is $1+\mathscr{F}(18)=6$. Hence (7) holds neither if (23) is considered as a left coset decomposition nor if it is considered as a right coset decomposition.

\section{References}

[1] M. Hall, Jr., The Theory of Groups, New York 1959.

[2] J. Mycielski et W. Sierpiński, Sur une propriété des ensembles linéaires, Fund. Math. 58 (1966), pp. 143-147. 
[3] S̆. Znám, On Mycielski's problem on system of arithmetic sequences, Colloquium Math. 15 (1966), pp. 201-204.

[4] - and I. Korec, On disjoint covering of groups by cosets by their subgroups (to appear).
Recu par la Rédaction le 6. 2.1973

\section{A new definition of the circle by the use of bisectors}

by

\author{
A. D. Berard, Jr. (Wilkes-Barre, Pa.) and W. Nitka (Oran)
}

Abstract. The subset $B(x, y)=\{q \in X: \varrho(x, q)=\varrho(y, q)\}$ in a metric space $(X, \varrho)$ is called the bisector of a pair $x, y$. It is known that any connected metric space in which each bisector is a unique point, is topologically an interval of the real line $R$.

If each bisector consists of exactly two points, then $X$ has DBP property.

The question whether every connected metric space with DBP is homeomorphic to the one-sphere $S^{1}$ is still open.

A metric space is segment-convex if for each pair $p, r$ of its points it contains an are joining $p$ to $r$ which is isometric to a line segment.

We show that any segment-convex metric space with DBP is isometric to a metric one-sphere with its natural geodesic metric.

1. Introduction. For any pair of distinct points $x$ and $y$ in a nontrivial metric space $(X, \varrho)$ the subset $B(x, y)=\{q \in X \mid \varrho(x, q)=\varrho(y, q)\}$ will be called the bisector ([3], see also [1] where it is called the midset). If each bisector is a unique point, then $X$ has [1] the unique bisector property (UBP). If each bisector consists of exactly two points, then $X$ has the double bisector property (DBP).

It is known [1] that any connected metric space with UBP is homeomorphic to a subset of the real line $R$, and is tnerefore an interval.

The question whether every connected metric space with DBP is homeomorphic to the one-sphere $S^{1}$ is still open.

The aim of the present paper is the following result: If $(X, \varrho)$ is a segment-convex metric space with $\mathrm{DBP}$, then $X$ is isometric to a metric one-sphere.

The proof will be based on the following three auxiliary propositions:

Let $a_{1}$ and $a_{2}$ be two distinct points of $X$, and let $B\left(a_{1}, a_{2}\right)$ $=\left\{x_{1}, x_{2}\right\}$, then

$1^{\circ} L_{1}=\overline{x_{1} a_{1}} \cup \overline{a_{1} x_{2}}$ and $L_{2}=\overline{x_{1} a_{2}} \cup \overline{a_{2} x_{2}}$ are two simple arcs joining $x_{1}$ to $x_{2}$, and $L_{1} \cap L_{2}=B\left(a_{1}, a_{2}\right)$.

$2^{\circ}$ More precisely, $L_{1}$ and $I_{2}$ are two metric segments joining $x_{1}$ to $x_{2}$.

$3^{\circ} I_{1} \cup L_{2}=X$. 